\title{
On Simple Exponential Sets of Polynomials
}

\author{
Z. G. Kishka, M. A. Saleem, and M. A. Abul-Dahab
}

\begin{abstract}
In this paper, it is shown that certain classes of regular functions of several complex variables be represented by exponential sets of polynomials in hyperelliptical regions. Moreover, an upper bound for the order of exponential set is given.
\end{abstract}

Mathematics Subject Classification (2000). 32A05, 32A17, 32A99.

Keywords. Basic sets of polynomials, exponential sets, hyperelliptical regions.

\section{Introduction}

One of the fundamental problems in the classical analytic function theory is the existence of bases. In complex analysis, the local behavior of an analytic function in an open disc $C(a, r)$ centered at $a$ and with radius $r$ is completely determined by means of a power series in $(z-a)$ for a fixed complex number $a$. The question whether the basic set (basis) of polynomials $\left\{(z-a)^{n}: n \in \mathbb{N}\right\}$ can be replaced by another set of polynomials (basis of polynomials) $\left\{P_{n}(z): n \in \mathbb{N}\right\}$, representing each $f(z)$ analytic in $C(a, r)$ has been a subject of intensive theoretical research in classical function theory and its applications. The main development in the subject towards its present state was due to Whittaker $[19,20]$ and Cannon $[7,8]$. Whittaker [20] laid down the minimum requirements for a set of polynomials to be suitable to represent a function and thus introduced the idea of "basic set" (or a basis) of polynomials. Consequently the expansion of a function by such series of polynomials is derived through an algebraic relation and not through a contour integral as in the previous trials. Also, considering the term function to refer to the Taylor expansion of the function about the origin, and thus reducing the domain of representation to a circle round the origin, Whittaker was able to introduce the idea of "effectiveness" of the set of polynomials and to obtain a criterion for effectiveness, i.e., a sufficient condition for effectiveness which does not depend on the particular function to be represented. This was proved to be necessary by Cannon [7] and thus the fundamental problem of the subject was solved. For sake of representation of entire (integral) functions, Whittaker introduced the idea of order and type of basic sets 
(or bases) of polynomials, by giving these sufficient conditions for the representation of integral (entire) functions of certain class. These conditions were also proved to be necessary by Cannon [8]. Furthermore, many properties of the "criterion" of effectiveness were discovered and the idea of representation near the origin was introduced and developed by Whittaker [20].

The study of the basic sets of polynomials of several complex variables was initiated by Mursi and Makar [15,16], Nassif [17], Kishka and others [9-14], where the representation in polycylindrical and hyperspherical regions was considered. Also, there are studies on basic sets of polynomials such as in Clifford analysis [1-6] and in Faber regions [18]. In this work, we aim to establish certain convergence properties of a new basic set of polynomials of several complex variables in hyperelliptical regions. This set will be called (exponential set) in which its matrices of coefficients and operators are $e^{p}$ and $e^{-p}$, respectively, with the properly that $e^{p} e^{-p}=I$. Also, effectiveness of this set is obtained. Moreover, the mode of increase of exponential set is given. For the purpose of this work, we give a different less elaborate exposition of the properties of theory polynomial bases. We refer the reader to $[11,12,14,17]$.

\section{Basic Sets of Polynomials of Several Complex Variables}

To avoid lengthy scripts, the following notations are adopted throughout this work (see $[11,12,17])$.

$$
\begin{aligned}
& \mathbf{m}=\left(m_{1}, m_{2}, \ldots, m_{k}\right) ;\langle\mathbf{m}\rangle=m_{1}+m_{2}+\cdots+m_{k} ; \\
& \mathbf{h}=\left(h_{1}, h_{2}, \ldots, h_{k}\right) ;\langle\mathbf{h}\rangle=h_{1}+h_{2}+\cdots+h_{k} ; \\
& \mathbf{z}=\left(z_{1}, z_{2}, \ldots, z_{k}\right) ; \quad \mathbf{z}^{\mathbf{m}}=z_{1}^{m_{1}} \cdot z_{2}^{m_{2}} \ldots . . z_{k}^{m_{k}} ; \quad \mathbf{0}=(0,0, \ldots, 0) ; \\
& |\mathbf{z}|^{2}=\left|z_{1}\right|^{2}+\left|z_{2}\right|^{2}+\cdots+\left|z_{k}\right|^{2} ; \quad \mathbf{t}^{\mathbf{m}}=t_{1}^{m_{1}} \cdot t_{2}^{m_{2}} \ldots . . t_{k}^{m_{k}} ; \\
& \mathbf{r}=\left(r_{1}, r_{2}, \ldots, r_{k}\right) ; \quad \mathbf{r}^{*}=\mathbf{r} \text { if } r_{s}=r \quad \forall s \in I ; \quad I=\{1,2,3, \ldots, k\} .
\end{aligned}
$$

In these notations, $m_{1}, m_{2}, \ldots, m_{k}$ and $h_{1}, h_{2}, \ldots, h_{k}$ are non-negative integers while $t_{1}, t_{2}, \ldots, t_{k}$ are non-negative numbers. Also, square brackets are used here in functional notation to express the fact that the function is either a function of several complex variables or one related to such function. In the space of several complex variables $\mathbb{C}^{k}$, an open hyperelliptical region $\sum_{s=1}^{k} \frac{\left|z_{s}\right|^{2}}{r_{s}^{2}}<1$ is here denoted by $\mathbf{E}_{[\mathbf{r}]}$ and its closure $\sum_{s=1}^{k} \frac{\left|z_{s}\right|^{2}}{r_{s}^{2}} \leq 1$, by $\overline{\mathbf{E}}_{[\mathbf{r}]}$, where $r_{s}, s \in I$ are positive numbers. In terms of the introduced notations, these regions satisfy the following inequalities:

$$
\begin{aligned}
& \mathbf{E}_{[\mathbf{r}]}=\{\mathbf{w}:|\mathbf{w}|<1\} \\
& \overline{\mathbf{E}}_{[\mathbf{r}]}=\{\mathbf{w}:|\mathbf{w}| \leq 1\},
\end{aligned}
$$

where $\mathbf{w}=\left(w_{1}, w_{2}, \ldots, w_{k}\right), w_{s}=\frac{z_{s}}{r_{s}} ; s \in I$. 
Suppose now that the function $f(\mathbf{z})$, is given by

$$
f(\mathbf{z})=\sum_{\mathbf{m}=\mathbf{0}}^{\infty} a_{\mathbf{m}} \mathbf{z}^{\mathbf{m}}
$$

is regular in $\overline{\mathbf{E}}_{[\mathbf{r}]}$ and

$$
M[f ;[\mathbf{r}]]=\sup _{\overline{\mathbf{E}}_{[\mathbf{r}]}}|f(\mathbf{z})|
$$

From (2.1) we easily see that $\left\{\left|z_{s}\right| \leq r_{s} t_{s}:|\mathbf{t}|=1\right\} \subset \overline{\mathbf{E}}_{[\mathbf{r}]}$, where $\mathbf{t}$ is the vector $\left(t_{1}, t_{2}, \ldots, t_{k}\right)$. Hence, it follows that

$$
\left|a_{\mathbf{m}}\right| \leq \sigma_{\mathbf{m}} \frac{M[f ;[\rho]]}{\Pi_{s=1}^{k}\left(\rho_{s}\right)^{m_{s}}},
$$

for all $0<\rho_{s}<r_{s} ; s \in I$, where

$$
\sigma_{\mathbf{m}}=\inf _{|t|=1} \frac{1}{t^{\mathbf{m}}}=\frac{\{\langle\mathbf{m}\rangle\}^{\frac{\langle\mathrm{m}\rangle}{2}}}{\Pi_{s=1}^{k} m_{s}^{\frac{m_{s}}{2}}}(\operatorname{see}[10,13]),
$$

and $1 \leq \sigma_{\mathbf{m}} \leq(\sqrt{n})^{\langle\mathbf{m}\rangle}$ on the assumption that $m_{s}^{\frac{m_{s}}{2}}=1$, whenever $m_{s}=$ $0 ; s \in I$.

Thus, it follows that

$$
\limsup _{\langle\mathbf{m}\rangle \rightarrow \infty}\left\{\frac{\left|a_{\mathbf{m}}\right|}{\sigma_{\mathbf{m}} \Pi_{s=1}^{k}\left(\rho_{s}\right)^{\langle\mathbf{m}\rangle-m_{s}}}\right\}^{\frac{1}{\langle\mathbf{m}\rangle}} \leq \frac{1}{\Pi_{s=1}^{k} \rho_{s}} .
$$

Since $\rho_{s}$ can be chosen arbitrarily near to $r_{s}, s \in I$, we conclude that

$$
\limsup _{\langle\mathbf{m}\rangle \rightarrow \infty}\left\{\frac{\left|a_{\mathbf{m}}\right|}{\sigma_{\mathbf{m}} \Pi_{s=1}^{k}\left(r_{s}\right)^{\langle\mathbf{m}\rangle-m_{s}}}\right\}^{\frac{1}{\langle\mathbf{m}\rangle}} \leq \frac{1}{\Pi_{s=1}^{k} r_{s}} .
$$

Then, it can be easily proved that the function $f(\mathbf{z})$ is regular in the open hyperelliptical $\mathbf{E}_{[\mathbf{r}]}$. The numbers $r_{s}$, given by (2.6), is thus conveniently called the radii of regularity of the function $f(\mathbf{z})$.

Definition 2.1. ([9,10]) A set of polynomials

$$
\left\{P_{\mathbf{m}}[\mathbf{z}]\right\}=\left\{P_{0}[\mathbf{z}], P_{1}[\mathbf{z}], P_{2}[\mathbf{z}], \ldots, P_{n}[\mathbf{z}], \ldots\right\},
$$

is said to be basic when every polynomial in the complex variables $z_{s}, s \in \mathrm{I}$, can be uniquely expressed as a finite linear combination of the elements of the set $\left\{P_{\mathbf{m}}[\mathbf{z}]\right\}$.

Thus, according to $[15]$ the set $\left\{P_{\mathbf{m}}[\mathbf{z}]\right\}$ will be basic if and and only if there exists a unique row-finite matrix $\bar{P}$ such that

$$
\bar{P} P=P \bar{P}=\mathbf{I},
$$

where $P=\left[P_{\mathbf{m} ; \mathbf{h}}\right]$ is the matrix of coefficients, $\bar{P}$ is the matrix of operators of the set $\left\{P_{\mathbf{m}}[\mathbf{z}]\right\}$ and $\mathbf{I}$ is the unit matrix. 
For the basic set $\left\{P_{\mathbf{m}}[\mathbf{z}]\right\}$ and its inverse $\left\{\bar{P}_{\mathbf{m}}[\mathbf{z}]\right\}$, we have

$$
\begin{gathered}
P_{\mathbf{m}}[\mathbf{z}]=\sum_{\mathbf{h}} P_{\mathbf{m} ; \mathbf{h}} \mathbf{z}^{\mathbf{h}}, \\
\bar{P}_{\mathbf{m}}[\mathbf{z}]=\sum_{\mathbf{h}} \bar{P}_{\mathbf{m} ; \mathbf{h}} \mathbf{z}^{\mathbf{h}}, \\
\mathbf{z}^{\mathbf{m}}=\sum_{\mathbf{h}} \bar{P}_{\mathbf{m} ; \mathbf{h}} P_{\mathbf{h}}[\mathbf{z}]=\sum_{\mathbf{h}} P_{\mathbf{m} ; \mathbf{h}} \bar{P}_{\mathbf{h}}[\mathbf{z}] .
\end{gathered}
$$

Thus, for the function $f(\mathbf{z})$ given in (2.2) we get

$$
f(\mathbf{z})=\sum_{\mathbf{m}} \Pi_{\mathbf{m}} P_{\mathbf{m}}[\mathbf{z}]
$$

where

$$
\Pi_{\mathbf{m}}=\sum_{\mathbf{h}} \bar{P}_{\mathbf{h} ; \mathbf{m}} a_{\mathbf{h}}=\sum_{\mathbf{h}} \bar{P}_{\mathbf{h} ; \mathbf{m}} \frac{f^{\mathbf{h}}(\mathbf{0})}{\mathbf{h} !},
$$

and $h !=h(h-1)(h-2) \ldots 3.2 .1$. The series $\sum_{\mathbf{m}}^{\infty} \Pi_{\mathbf{m}} P_{\mathbf{m}}[\mathbf{z}]$ is the associated basic series of $f(\mathbf{z})$.

Definition 2.2. ([10,11]) The associated basic series $\sum_{\mathbf{m}}^{\infty} \Pi_{\mathbf{m}} P_{\mathbf{m}}[\mathbf{z}]$ is said to represent $f(\mathbf{z})$ in

(i) $\overline{\mathbf{E}}_{[\mathbf{r}]}$ when it converges uniformly to $f(\mathbf{z})$ in $\overline{\mathbf{E}}_{[\mathbf{r}]}$,

(ii) $\mathbf{E}_{[\mathbf{r}]}$ when it converges uniformly to $f(\mathbf{z})$ in $\mathbf{E}_{[\mathbf{r}]}$,

(iii) $\mathbf{D}\left(\overline{\mathbf{E}}_{[\mathbf{r}]}\right)$ when it converges uniformly to $f(\mathbf{z})$ in some hyperelliptical surrounding the hyperelliptical $\overline{\mathbf{E}}_{[\mathbf{r}]}$, not necessarily the former hyperelliptical.

Definition 2.3. ([11,12]) The set $\left\{P_{\mathbf{m}}[\mathbf{z}]\right\}$ is said to be simple set, when the polynomial $P_{\mathbf{m}}[\mathbf{z}]$ is of degree $\langle\mathbf{m}\rangle$, that is to say

$$
P_{\mathbf{m}}[\mathbf{z}]=\sum_{(\mathbf{h})=\mathbf{0}}^{(\mathbf{m})} P_{\mathbf{m} ; \mathbf{h}} \mathbf{z}^{\mathbf{h}} .
$$

A simple set $\left\{P_{\mathbf{m}}[\mathbf{z}]\right\}$ is said to be absolutely monic if the coefficient $P_{\mathbf{m}, \mathbf{m}}$ of $z_{1}^{m_{1}} z_{2}^{m_{2}} \ldots z_{s}^{m_{s}}$ in (2.13) is unity.

Definition 2.4. ([11,12]) Let $N_{\mathbf{m}}=N_{m_{1}, m_{2}, \ldots, m_{n}}$ be the number of non-zero coefficients $\bar{P}_{\mathbf{m} ; \mathbf{h}}$ in the representation (2.9). A basic set satisfying the condition

$$
\lim _{\langle\mathbf{m}\rangle}\left\{N_{\mathbf{m}}\right\}^{\frac{1}{\langle\mathbf{m}\rangle}}=1,
$$

is called a Cannon set and if

$$
\lim _{\langle\mathbf{m}\rangle}\left\{N_{\mathbf{m}}\right\}^{\frac{1}{\langle\mathbf{m}\rangle}}=a>1,
$$

then the set is called a general basic set. 
Now, let $D_{\mathbf{m}}=D_{m_{1}, m_{2}, \ldots, m_{n}}$ be the degree of the polynomial of the highest degree $\mathrm{n}$ represented in (2.9), that is to say, if $D_{\mathbf{h}}=D_{h_{1}, h_{2}, \ldots, h_{n}}$ is the degree of the polynomial $P_{\mathbf{m}}$, then $D_{\mathbf{h}}<D_{\mathbf{m}} \forall h_{s}<m_{s}$. Since the elements of the basic set are linearly independent, then $N_{\mathbf{m}} \leq 1+2+3+\cdots+\left(D_{\mathbf{m}+1}\right) \leq$ $\lambda_{1} D_{\mathbf{m}}^{2}$, where $\lambda_{1}$ is a constant. Therefore, the conditions (2.14) for a basic set to be a Cannon set implies the following condition (see $[10,11])$ :

$$
\lim _{\langle\mathbf{m}\rangle \rightarrow \infty}\left\{D_{\mathbf{m}}\right\}^{\frac{1}{\langle\mathbf{m}\rangle}}=1 .
$$

For any function $f(\mathbf{z})$ of several complex variables, there is formally an associated basic series $\sum_{\mathbf{h}=\mathbf{0}}^{\infty} \Pi_{\mathbf{h}} P_{\mathbf{h}}[\mathbf{z}]$. When this associated series converges uniformity to $f(\mathbf{z})$ in some domain it is said to represent $f(\mathbf{z})$ in that domain. In other words, as in the classical terminology of Whittaker (see [19,20]), the basic set $\left\{P_{\mathbf{m}}[\mathbf{z}]\right\}$ will be effective in that domain. The convergence properties of basic sets of polynomials are classified according to the classes of functions represented by their associated basic series and also to the domain in which they are represented.

To study the convergence properties of such basic sets of polynomials in hyperelliptical regions (c.f. $[10,11]$ ), we consider the following notations for Cannon sums:

$$
\Omega\left[P_{\mathbf{m}}, \overline{\mathbf{E}}_{[\mathbf{r}]}\right]=\sigma_{\mathbf{m}} \Pi_{s=1}^{n}\left\{r_{s}\right\}^{\langle\mathbf{m}\rangle-m_{s}} \sum_{\mathbf{h}}\left|\bar{P}_{\mathbf{m}, \mathbf{h}}\right| M\left(P_{\mathbf{m}}, \overline{\mathbf{E}}_{[\mathbf{r}]}\right) .
$$

Also, the Cannon function for the basic sets of polynomials in hyperelliptical regions was defined as follows:

$$
\Omega\left[P, \overline{\mathbf{E}}_{[\mathbf{r}]}\right]=\limsup _{\langle\mathbf{m}\rangle \rightarrow \infty}\left\{\Omega\left[P_{\mathbf{m}}, \overline{\mathbf{E}}_{[\mathbf{r}]}\right]\right\}^{\frac{1}{\langle\mathbf{m}\rangle}} .
$$

Concerning the effectiveness of the basic set of polynomials of several complex variables in hyperelliptical regions, we have from $[10,11]$ the following results.

Theorem 2.1. The necessary and sufficient condition for the Cannon basic set $\left\{P_{\mathbf{m}}[\mathbf{z}]\right\}$ of polynomials of several complex variables to be effective in the closed hyperellipse $\overline{\mathbf{E}}_{[\mathbf{r}]}$ is that $\Omega\left[P, \overline{\mathbf{E}}_{[\mathbf{r}]}\right]=\prod_{s=1}^{n} r_{s}$.

\section{Effectiveness of Exponential Sets}

Let $\left\{\mathbb{P}_{\mathbf{m}}(\mathbf{z})\right\}$ be the exponential set of polynomials of the several complex variables $z_{1}, z_{2}, \ldots, z_{s}$, whose matrices of coefficients and operators are $\mathbf{e}^{\mathbf{P}}$ and $\mathbf{e}^{-\mathbf{P}}$, respectively. Thus $\mathbf{e}^{\mathbf{P}} \mathbf{e}^{-\mathbf{P}}=\mathbf{I}$, and the exponential set $\left\{\mathbb{P}_{\mathbf{P}}(\mathbf{z})\right\}$ is basic, associated with the simple monic set $\left\{\mathbb{P}_{\mathbf{m}}(\mathbf{z})\right\}$.

Suppose that the elements $\mathbf{P}_{\mathbf{m} ; \mathbf{h}}$ of the matrix of coefficients of the set $\left\{\mathbb{P}_{\mathbf{m}}(\mathbf{z})\right\}$ satisfy the following inequality

$$
\left|\mathbf{P}_{\mathbf{m} ; \mathbf{h}}\right| \leq M \frac{\sigma_{\mathbf{h}} \Pi_{s=1}^{k} a_{s}^{m_{s}-h_{s}}}{\sigma_{\mathbf{m}}[(\mathbf{m})+1]} ; \quad \mathbf{0} \leq(\mathbf{h})<(\mathbf{m})
$$


where $M \geq 1$ and $a_{s}>0$. Then, for a positive integral $j \geq 1$, if $\mathbf{P}_{\mathbf{m} ; \mathbf{h}}^{j}$ are the elements of the power matrix $\mathbf{P}^{j}$, it follows that

$$
\mathbf{P}_{\mathbf{m} ; \mathbf{h}}^{j}=\sum_{(\mathbf{s})=(\mathbf{h})}^{(\mathbf{m})} \mathbf{P}_{\mathbf{m} ; \mathbf{s}} \mathbf{P}_{\mathbf{s} ; \mathbf{h}}^{j-1}
$$

First put $j=2$ in (3.2) and apply (3.1) to get

$$
\begin{aligned}
\mathbf{P}_{\mathbf{m} ; \mathbf{h}}^{(2)} & \leq M^{2} \Pi_{s=1}^{k} a_{s}^{m_{s}-h_{s}} \frac{\sigma_{\mathbf{h}}}{\sigma_{\mathbf{m}}[(\mathbf{m})+1]} \sum_{(\mathbf{s})=(\mathbf{h})}^{(\mathbf{m})} \frac{1}{(\mathbf{s})+1} \\
& <M^{2} \Pi_{s=1}^{k} a_{s}^{m_{s}-h_{s}} \frac{\sigma_{\mathbf{h}}}{\sigma_{\mathbf{m}}}, \quad \mathbf{0} \leq(\mathbf{h})<(\mathbf{m}),
\end{aligned}
$$

we shall assume that

$$
\left|\mathbf{P}_{\mathbf{m} ; \mathbf{h}}^{j}\right| \leq M^{j} \Pi_{s=1}^{k} a_{s}^{m_{s}-h_{s}} \frac{\sigma_{\mathbf{h}}}{\sigma_{\mathbf{m}}}, \quad \mathbf{0} \leq(\mathbf{h})<(\mathbf{m}) .
$$

Applying (3.1), (3.2) and (3.3), we have

$$
\left|\mathbf{P}_{\mathbf{m} ; \mathbf{h}}^{(j+1)}\right|=\left|\sum_{(\mathbf{s})=(\mathbf{h})}^{(\mathbf{m})} \mathbf{P}_{\mathbf{m} ; \mathbf{s}} \mathbf{P}_{\mathbf{s} ; \mathbf{h}}^{j}\right| \leq M^{j+1} \Pi_{s=1}^{k} a_{s}^{m_{s}-h_{s}} \frac{\sigma_{\mathbf{h}}}{\sigma_{\mathbf{m}}} .
$$

Hence, the assumption (3.3) is justified by mathematical induction.

Since $\mathbf{e}^{\mathbf{P}}=E_{\mathbf{m} ; \mathbf{h}}$ is the matrix of coefficients of the exponential set $\left\{\mathbb{P}_{\mathbf{m}}(\mathbf{z})\right\}$, then it follows that

$$
\mathbb{P}_{\mathbf{m}}(\mathbf{z})=\sum_{(\mathbf{h})=\mathbf{0}}^{\mathbf{m}} E_{\mathbf{m} ; \mathbf{h}} \mathbf{z}^{\mathbf{h}}=\sum_{(\mathbf{h})=\mathbf{0}}^{(\mathbf{m})}\left\{\sum_{j=0}^{\infty} \frac{\mathbf{P}_{\mathbf{m} ; \mathbf{h}}^{(j)}}{j !}\right\} \mathbf{z}^{\mathbf{h}}
$$

We can write

$$
\begin{aligned}
\mathbf{M}\left(\mathbb{P}_{\mathbf{m}} ; \overline{\mathbf{E}}_{[\mathbf{r}]}\right) & =\sup _{\overline{\mathbf{E}}_{[\mathbf{r}]}}\left|\mathbb{P} \mathbb{E}_{\mathbf{m}}(\mathbf{z})\right| \leq \sum_{(\mathbf{h})=\mathbf{0}}^{(\mathbf{m})} \sum_{j=0}^{\infty} \frac{M^{j} \Pi_{s=1}^{k} a_{s}^{m_{s}-h_{s}}}{j !} \frac{r_{s}^{h_{s}}}{\sigma_{\mathbf{m}}} \\
& =\frac{e^{M}}{\sigma_{\mathbf{m}}} \sum_{(\mathbf{h})=\mathbf{0}}^{(\mathbf{m})} \Pi_{s=1}^{k}\left(\frac{a_{s}}{r_{s}}\right)^{m_{s}-h_{s}} r_{s}^{m_{s}} \\
& \leq \frac{e^{M}}{\sigma_{\mathbf{m}}}\{(\mathbf{m})+1\} \Pi_{s=1}^{k} r_{s}^{m_{s}} \forall r_{s} \geq a_{s} .
\end{aligned}
$$

Also, since the matrix of operator of the exponential set $\left\{\mathbb{P}_{\mathbf{m}}(\mathbf{z})\right\}$ is $\mathbf{e}^{-\mathbf{P}}=$ $\bar{E}_{\mathbf{m} ; \mathbf{h}}$, hence as an application of (3.3) and (3.6) in the Cannon sum $\Omega\left[\mathbb{P} \mathbb{E}_{\mathbf{m}} ; \overline{\mathbf{E}}_{[\mathbf{r}]}\right]$ 
of the simple set $\left\{\mathbb{P}_{\mathbf{P}}(\mathbf{z})\right\}$ for the closed hyperelliptical $\overline{\mathbf{E}}_{[\mathbf{r}]}$ leads to

$$
\begin{aligned}
\Omega\left[\mathbb{P} \mathbb{E}_{\mathbf{m}} ; \overline{\mathbf{E}}_{[\mathbf{r}]}\right] & =\sigma_{\mathbf{m}} \Pi_{s=1}^{k} r_{s}^{\langle\mathbf{m}\rangle-m_{s}} \sum_{(\mathbf{h})=\mathbf{0}}^{(\mathbf{m})}\left|\bar{E}_{\mathbf{m} ; \mathbf{h}}\right| \mathbf{M}\left(\mathbb{P}_{\mathbf{m}} ; \overline{\mathbf{E}}_{[\mathbf{r}]}\right) \\
& =\sigma_{\mathbf{m}} \Pi_{s=1}^{k} r_{s}^{\langle\mathbf{m}\rangle-m_{s}} \sum_{(\mathbf{h})=\mathbf{0}}^{(\mathbf{m})}\left|\sum_{j=0}^{\infty}(-1)^{j} \frac{\mathbf{P}_{\mathbf{m} ; \mathbf{h}}^{(j)}}{j !}\right| \mathbf{M}\left(\mathbb{P}_{\mathbb{P}} \mathbb{E}_{\mathbf{m}} ; \overline{\mathbf{E}}_{[\mathbf{r}]}\right) \\
& <e^{2 M} \Pi_{s=1}^{k} r_{s}^{\langle\mathbf{m}\rangle-m_{s}} \sum_{(\mathbf{h})=\mathbf{0}}^{(\mathbf{m})} a_{s}^{m_{s}-h_{s}} r_{s}^{h_{s}}[(\mathbf{h})+1] \\
& <e^{2 M}[(\mathbf{m})+1]^{2} \Pi_{s=1}^{k} r_{s}^{\langle\mathbf{m}\rangle} \forall r_{s} \geq a_{s} .
\end{aligned}
$$

Now, the Cannon function of the exponential set $\left\{\mathbb{P}_{\mathbf{m}}(\mathbf{z})\right\}$ for the closed hyperelliptical $\left.\overline{\mathbf{E}}_{[\mathbf{r}]}\right]$ given by

$$
\Omega\left[\mathbb{P} \mathbb{E} ; \overline{\mathbf{E}}_{[\mathbf{r}]}\right]=\limsup _{\langle\mathbf{m}\rangle \rightarrow \infty}\left\{\Omega\left[\mathbb{P}_{\mathbb{m}} ; \overline{\mathbf{E}}_{[\mathbf{r}]}\right]\right\}^{\frac{1}{\langle\mathbf{m}\rangle}} \leq \Pi_{s=1}^{k} r_{s}, \quad \forall r_{s} \geq a_{s} .
$$

Since $\left\{\mathbb{P} \mathbb{E}_{\mathbf{m}}(\mathbf{z})\right\}$ is basic, then there exists a unique representation in the form

$$
\mathbf{z}^{\mathbf{m}}=\Pi_{s=1}^{k} z_{s}^{m_{s}}=\sum_{(\mathbf{h})=\mathbf{0}}^{(\mathbf{m})} \bar{E}_{\mathbf{m} ; \mathbf{h} \mathbb{P}} \mathbb{E}_{\mathbf{m}}(\mathbf{z}),
$$

from this, we obtain

$$
\begin{aligned}
& \sigma_{\mathbf{m}} \Pi_{s=1}^{k} r_{s}^{\langle\mathbf{m}\rangle-m_{s}} \sup _{\overline{\mathbf{E}}_{[\mathrm{r}]}}\left|z_{2}^{m_{2}} z_{k}^{m_{k}}\right|=\Pi_{s=1}^{k} r_{s}^{\langle\mathbf{m}\rangle} \\
& \leq \sigma_{\mathbf{m}} \Pi_{s=1}^{k} r_{s}^{\langle\mathbf{m}\rangle-m_{s}} \sup _{\overline{\mathbf{E}}_{[\mathrm{r}]}} \sum_{(\mathbf{h})=\mathbf{0}}^{(\mathbf{m})}\left|\bar{E}_{\mathbf{m} ; \mathbf{h}}\right| \mathbf{M}\left(\mathbb{P}_{\mathbf{m}} ; \overline{\mathbf{E}}_{[\mathbf{r}]}\right) \\
&=\Omega\left[\mathbb{P}_{\mathbf{m}} ; \overline{\mathbf{E}}_{[\mathbf{r}]}\right],
\end{aligned}
$$

that is to say

$$
\Omega\left[\mathbb{P} \mathbb{E} ; \overline{\mathbf{E}}_{[\mathbf{r}]}\right] \geq \Pi_{s=1}^{k} r_{s} .
$$

It follows from (3.7) and (3.8), we see that

$$
\Omega\left[\mathbb{P} \mathbb{E} ; \overline{\mathbf{E}}_{[\mathbf{r}]}\right]=\Pi_{s=1}^{k} r_{s}, \text { for all } r_{s} \geq a_{s} ; s \in I .
$$

Therefore, we infer that the exponential set $\left\{\mathbb{P}_{\mathbf{m}}(\mathbf{z})\right\}$ is effective in the closed hyperellipse $\overline{\mathbf{E}}_{[\mathbf{r}]}$, for all $r_{s} \geq a_{s} ; s \in I$.

Remark 3.1. When $r_{s}<a_{s}, s \in I$, the exponential set $\left\{\mathbb{P}_{\mathbf{m}}(\mathbf{z})\right\}$ may not be effective in $\overline{\mathbf{E}}_{[\mathbf{r}]}$. This fact is shown by the following example:

Example 3.1. Consider the simple monic set $\mathbf{p}_{\mathbf{m}}(\mathbf{z})$ for which

$$
\mathbf{p}_{\mathbf{m} ; \mathbf{h}}=\left\{\begin{array}{l}
1 ; \quad(\mathbf{h})=(\mathbf{m}), \\
M \frac{\sigma_{\mathbf{h}} a^{\langle\mathbf{m}\rangle-\langle\mathbf{h}\rangle}}{\sigma_{\mathbf{m}}[(\mathbf{m})+1]} ; \quad a>0, \quad \mathbf{0} \leq(\mathbf{h})<(\mathbf{m}) .
\end{array}\right.
$$


As in (3.3) we can apply (3.9) to prove by mathematical induction that

$$
\mathbf{p}_{\mathbf{m} ; \mathbf{0}}^{(j)} \geq \frac{M^{j}}{\sigma_{\mathbf{m}}[(\mathbf{m})+1]} a^{\langle\mathbf{m}\rangle} ; \quad j \geq 1
$$

Thus, the Cannon sum of the the exponential set $\left\{\mathbb{P} \mathbb{E}_{\mathbf{m}}(\mathbf{z})\right\}$ for the equihyperellipse $\overline{\mathbf{E}}_{[\mathrm{r}] *}$ will be such that

$$
\begin{aligned}
\Omega\left[\mathbb{P} \mathbb{E}_{\mathbf{m}} ; \overline{\mathbf{E}}_{[\mathbf{r}]^{*}}\right] & =\sigma_{\mathbf{m}} \Pi_{s=1}^{k} r_{s}^{\langle\mathbf{m}\rangle-m_{s}} \sum_{(\mathbf{h})=0}^{(\mathbf{m})}\left|\bar{E}_{\mathbf{m} ; \mathbf{h}}\right| \mathbf{M}\left(\mathbb{P}_{\mathbf{m}} ; \overline{\mathbf{E}}_{[\mathbf{r}]^{*}}\right) \\
& \geq \sigma_{\mathbf{m}} r^{(k-1)\langle\mathbf{m}\rangle}\left|\bar{E}_{\mathbf{m} ; \mathbf{m}}\right| \mathbf{M}\left(\mathbb{P} \mathbb{E}_{\mathbf{m}} ; \overline{\mathbf{E}}_{[\mathbf{r}]^{*}}\right) \\
& =\sigma_{\mathbf{m}} r^{(k-1)\langle\mathbf{m}\rangle}\left|\sum_{j=0}^{\infty} \frac{(-1)^{j}}{j !} \mathbf{p}_{\mathbf{m} ; \mathbf{m}}^{(j)}\right| \mathbf{M}\left(\mathbb{P} \mathbb{E}_{\mathbf{m}} ; \overline{\mathbf{E}}_{[\mathbf{r}]^{*}}\right) \\
& \geq \sigma_{\mathbf{m}} r^{(k-1)\langle\mathbf{m}\rangle} e^{-1}\left|E_{\mathbf{m} ; \mathbf{0}}\right|=\sigma_{\mathbf{m}} r^{(k-1)\langle\mathbf{m}\rangle} e^{-1}\left|\sum_{j=0}^{\infty} \frac{\mathbf{p}_{\mathbf{m} ; 0}^{(j)}}{j !}\right| \\
& >\sigma_{\mathbf{m}} r^{(k-1)\langle\mathbf{m}\rangle} e^{-1} \frac{a^{\langle\mathbf{m}\rangle}}{\sigma_{\mathbf{m}}[(\mathbf{m})+1]} \sum_{j=1}^{\infty} \frac{M^{j}}{j !} \\
& =\frac{e^{-1}\left[e^{M}-1\right]}{(\mathbf{m})+1}\left[r^{(k-1)} a\right]^{\langle\mathbf{m}\rangle} \cdot
\end{aligned}
$$

Thus, $\Omega\left[\mathbb{P} \mathbb{E} ; \overline{\mathbf{E}}_{\left.[\mathrm{r}]^{*}\right]} \geq r^{(k-1)} a\right.$ for all $r<a$, and the exponential set $\left\{\mathbb{P} \mathbb{E}_{\mathbf{m}}(\mathbf{z})\right\}$ is not effective in $\overline{\mathbf{E}}_{[\mathrm{r}] *}$ for $r<a$ as required.

Therefore, the following theorem is completely established.

Theorem 3.1. If $\left\{\mathbb{P}_{\mathbf{m}}(\mathbf{z})\right\}$ is a simple monic set satisfying (3.1), then the exponential set $\left\{\mathbb{P} \mathbb{E}_{\mathbf{m}}(\mathbf{z})\right\}$ is effective in $\overline{\mathbf{E}}_{[\mathbf{r}]}$, for all $r_{s} \geq a_{s} ; s \in I$ and may not be effective in $\overline{\mathbf{E}}_{[\mathrm{r}]}$ for $r_{s}<a_{s} ; s \in I$.

\section{Mode of Increase of Exponential Set}

The mode of increase of a base $P_{n}(z)$ is determined by the order and type of the base. For a simple base $\left\{P_{n}(z)\right\}$, the order $\rho$ is defined by [20]

$$
\rho=\lim _{r \rightarrow \infty} \limsup _{n \rightarrow \infty} \frac{\log \omega_{n}(r)}{n \log n},
$$

where $\omega_{n}(r)$ stands as usual for the Cannon sum, given above. If $0<\rho<1$, the type $\tau$ is given by

$$
\tau=\limsup _{r \rightarrow \infty} \frac{e}{\rho} \limsup _{n \rightarrow \infty} \frac{\left[\omega_{n}(r)\right]^{\frac{1}{n \rho}}}{n} .
$$

It has been shown in [17] that the upper bound of the class of entire functions of several complex variables represented by a given base is determined by the mode of increase of the base. The significance of the order and type of a base 
lies in the fact that they define the class of entire functions represented by the base. Thus, the problem to be investigated in this section is that the mode of increase of the exponential set $\left\{\mathbb{P}_{\mathbf{m}}(\mathbf{z})\right\}$ in the equi-hyperellipse $\overline{\mathbf{E}}_{[\mathbf{r}]^{*}}$.

Suppose that $\left\{\mathbb{P}_{\mathbf{m}}(\mathbf{z})\right\}$ is a simple monic set for which

$$
\mathbf{p}_{\mathbf{m} ; \mathbf{h}}=M \frac{\langle\mathbf{m}\rangle^{\lambda(\langle\mathbf{m}\rangle-\langle\mathbf{h}\rangle)}}{[(\mathbf{m})+1]} ; \quad \mathbf{0} \leq(\mathbf{h})<(\mathbf{m}),
$$

where $\lambda$ is positive constant and $M \geq 1$, is finite number.

Applying (3.1) in (4.1), it can be easily seen by induction that

$$
\left|\mathbf{p}_{\mathbf{m} ; \mathbf{h}}^{j}\right|<M^{j}\langle\mathbf{m}\rangle^{\lambda(\langle\mathbf{m}\rangle-\langle\mathbf{h}\rangle)} ; \quad(\mathbf{h})<(\mathbf{m}) .
$$

Thus, for the maximum modulus $\mathbf{M}\left(\mathbb{P}_{\mathbb{P}} ; \overline{\mathbf{E}}_{[\mathbf{r}]^{*}}\right)$ of the exponential set $\left\{\mathbb{P}_{\mathbf{m}}(\mathbf{z})\right\}$ we have

$$
\begin{aligned}
& \mathbf{M}\left({ }_{\mathbb{P}} \mathbb{E}_{\mathbf{m}} ; \overline{\mathbf{E}}_{[\mathbf{r}]^{*}}\right)=\sup _{\overline{\mathbf{E}}_{[\mathrm{r}]^{*}}}\left|\mathbb{P}_{\mathbf{m}}(\mathbf{z})\right| \leq \sum_{(\mathbf{h})=0}^{(\mathbf{m})} \sum_{j=0}^{\infty}\left|\frac{\mathbf{p}_{\mathbf{m} ; \mathbf{h}}^{(j)}}{j !}\right| \frac{r^{\langle\mathbf{h}\rangle}}{\sigma_{\mathbf{h}}} \\
& \leq \frac{e^{M}}{(\mathbf{m})+1} \sum_{(\mathbf{h})=\mathbf{0}}^{(\mathbf{m})}\left(\frac{r^{\frac{1}{\lambda}}}{\langle\mathbf{m}\rangle}\right)^{\lambda\langle\mathbf{h}\rangle}\langle\mathbf{m}\rangle^{\lambda\langle\mathbf{m}\rangle} \\
& <e^{M}\langle\mathbf{m}\rangle^{\lambda\langle\mathbf{m}\rangle}, \text { for }\langle\mathbf{m}\rangle^{\lambda}>r \text {. }
\end{aligned}
$$

Introducing (4.2) and (4.3) in the Cannon sum $\Omega\left[\mathbb{P}_{\mathbf{m}} ; \overline{\mathbf{E}}_{[\mathbf{r}]^{*}}\right]$ of the exponential set $\left\{\mathbb{P}_{\mathbf{m}}(\mathbf{z})\right\}$ we find

$$
\begin{aligned}
& \Omega\left[\mathbb{P} \mathbb{E}_{\mathbf{m}} ; \overline{\mathbf{E}}_{\left.[\mathbf{r}]^{*}\right]}=\sigma_{\mathbf{m}} \sum_{(\mathbf{h})=\mathbf{0}}^{(\mathbf{m})}\left|\bar{E}_{\mathbf{m} ; \mathbf{h}}\right| \mathbf{M}\left(\mathbb{P}_{\mathbb{h}} ; \overline{\mathbf{E}}_{[\mathbf{r}]^{*}}\right)\right. \\
& =\sigma_{\mathbf{m}} \sum_{(\mathbf{h})=\mathbf{0}}^{(\mathbf{m})} \sum_{j=0}^{\infty}\left|\frac{(-1)^{j} \mathbf{p}_{\mathbf{m} ; \mathbf{h}}^{(j)}}{j !}\right| \mathbf{M}\left(\mathbb{P} \mathbb{E}_{\mathbf{h}} ; \overline{\mathbf{E}}_{[\mathbf{r}]^{*}}\right) \\
& <e^{2 M} \sigma_{\mathbf{m}}|\mathbf{m}\rangle^{\lambda\langle\mathbf{m}\rangle}<(\sqrt{2})(\langle\mathbf{m}\rangle) e^{2 M}\langle\mathbf{m}\rangle^{\lambda\langle\mathbf{m}\rangle} .
\end{aligned}
$$

Thus, the order $\Gamma$ of the exponential set $\left\{\mathbb{P} \mathbb{E}_{\mathbf{m}}(\mathbf{z})\right\}$ will be such that

$$
\Gamma=\lim _{r \rightarrow \infty} \limsup _{\langle\mathbf{m}\rangle \rightarrow \infty} \frac{\log \Omega\left[\mathbb{P} \mathbb{E}_{\mathbf{m}} ; \overline{\mathbf{E}}_{\left.[\mathbf{r}]^{*}\right]}\right.}{\langle\mathbf{m}\rangle \log \langle\mathbf{m}\rangle} \leq \lambda .
$$

The fact that the bound $\lambda$ is attainable is illustrated by the following example:

Example 4.1. Consider the set $\left\{P_{\mathbf{m}}(\mathbf{z})\right\}$ given by

$$
\mathbf{p}_{\mathbf{m} ; \mathbf{h}}=\left\{\begin{array}{l}
1 ; \quad(\mathbf{h})=(\mathbf{m}), \\
M \frac{\langle\mathbf{m}\rangle^{\lambda(\langle\mathbf{m}\rangle-\langle\mathbf{h}\rangle)}}{[(\mathbf{m})+1]} ; \quad \mathbf{0} \leq(\mathbf{h})<(\mathbf{m}) .
\end{array}\right.
$$

As in (4.5) we can apply (3.10) to prove by induction that

$$
\mathbf{p}_{\mathbf{m} ; \mathbf{0}}^{(j)}>M \frac{\langle\mathbf{m}\rangle^{\lambda\langle\mathbf{m}\rangle}}{(\mathbf{m})+1} ; \quad j \geq 2 .
$$


Thus, the Cannon sum $\Omega\left[\mathbb{P} \mathbb{E}_{\mathbf{m}} ; \overline{\mathbf{E}}_{[\mathbf{r}]^{*}}\right]$ of the exponential set $\left\{\mathbb{P}_{\mathbf{m}}(\mathbf{z})\right\}$ will be such that

$$
\begin{aligned}
\Omega\left[\mathbb{P} \mathbb{E}_{\mathbf{m}} ; \overline{\mathbf{E}}_{\left.[\mathbf{r}]^{*}\right]}\right. & =\sigma_{\mathbf{m}} \sum_{(\mathbf{h})=\mathbf{0}}^{(\mathbf{m})}\left|\bar{E}_{\mathbf{m} ; \mathbf{h}}\right| \mathbf{M}\left(\mathbb{P}_{\mathbf{h}} ; \overline{\mathbf{E}}_{[\mathbf{r}]^{*}}\right) \\
& \geq \sigma_{\mathbf{m}}\left|\bar{E}_{\mathbf{m} ; \mathbf{h}}\right| \mathbf{M}\left(\mathbb{P}_{\mathbb{P}} \mathbb{E}_{\mathbf{h}} ; \overline{\mathbf{E}}_{[\mathbf{r}]^{*}}\right)=\sigma_{\mathbf{m}} e^{-1} \mathbf{M}\left(\mathbb{P}_{\mathbb{h}} ; \overline{\mathbf{E}}_{[\mathbf{r}]^{*}}\right) \\
& \geq e^{-1}\left|\bar{E}_{\mathbf{m} ; \mathbf{0}}\right| \geq e^{-1} \frac{\langle\mathbf{m}\rangle^{\lambda\langle\mathbf{m}\rangle}}{[(\mathbf{m})+1]} .
\end{aligned}
$$

So that,

$$
\Gamma=\lim _{r \rightarrow \infty} \limsup _{\langle\mathbf{m}\rangle \rightarrow \infty} \frac{\log \Omega\left[\mathbb{P} \mathbb{E}_{\mathbf{m}} ; \overline{\mathbf{E}}_{\left.[\mathbf{r}]^{*}\right]}\right.}{\langle\mathbf{m}\rangle \log \langle\mathbf{m}\rangle} \geq \lambda .
$$

Therefore, from (4.4) and (4.6), we conclude that $\Gamma=\lambda$ and the bound is thus attainable. This completes the proof of the following theorem:

Theorem 4.1. When condition (4.1) is satisfied, then the exponential set $\mathbb{P}_{\mathbf{m}}(\mathbf{z})$ will be of order $\Gamma \leq \lambda$. Moreover, the value $\lambda$ is attainable.

\section{Acknowledgment}

The authors express their sincere appreciation to Professor H. Begehr for valuable discussion, suggestions and comments.

Open Access. This article is distributed under the terms of the Creative Commons Attribution License which permits any use, distribution, and reproduction in any medium, provided the original author(s) and the source are credited.

\section{References}

[1] Abul-Ez, M.: Inverse sets of polynomials in Clifford analysis. Arch. der Math. 58, 561-567 (1992)

[2] Abul-Ez, M., Constales, D.: Similar functions and similar bases of polynomials in Clifford setting. Complex Variables 48, 1055-1070 (2003)

[3] Abul-Ez, M., Saleem, M., Zayed, M.: On the representation near a point of Clifford valued functions by infinite series of polynomials. In: 9th International Conferenceon Clifford Algebras, Weimar, Germany, 15-20 July (2011)

[4] Abul-Ez, M., Morais, J., Zayed, M.: Generalized derivative and primitive of Cliffordian bases of polynomials constructed through Appell monomials. Comput. Meth. Func. Theo. 4, 501-515 (2012)

[5] Abul-Ez, M., Saleem, M., Abd-Elmageed, H., Morais, J.: On polynomial series expansions of Cliffordian functions in open balls and at the origin. Cliff. Anal. Cliff. Alge. Appl. 2, 291-307 (2012)

[6] Abul-Ez, M., Constales, D.: On the order of basic series representing Clifford valued functions. Appl. Math. Comput. 142, 575-584 (2003)

[7] Cannon, B.: On the convergence of series of polynomials. Proc. Lond. Math. Soc. 43, 348-364 (1937)

[8] Cannon, B.: On the representation of integral functions by general basic series. Math. Zeit. 45, 185-208 (1939) 
[9] El-Sayed, A.: On derived and integrated sets of basic sets of polynomials of several complex variables. Acta Math. Acad. Paed. Nyiregyhazi 19, 195-204 (2003)

[10] El-Sayed, A., Kishka, Z.M.G.: On the effectiveness of basic sets of polynomials of several complex variables in elliptical regions. In: Proceedings of the 3rd International ISAAC Congress, pp. 265-278. Freie Universitaet Berlin, Germany. Kluwer, Dordrecht (2003)

[11] Hassan, G.F.: Ruscheweyh differential operator sets of basic sets of polynomials of several complex variables in hyperelliptical regions. Acta Math. Acad. Paed. Nyiregyhazi 22, 247-264 (2006)

[12] Kishka, Z.M.G.: On the convergence of properties of basic sets of polynomials of several complex variables i. Sohg Pure, Appl. Sci. Bull. Fac. Sci. Assiut Univ. 7, 71-119 (1991)

[13] Kishka, Z.M.G.: Power set of simple set of polynomials of two complex variables. Bull. Soc. R. Sci. Liege 62, 265-278 (1993)

[14] Kishka, Z.M.G, El-Sayed, A.: On the order and type of basic and composite sets of polynomials in complete Reinhardt domains. Period. Math. Hung. 46, 67$79(2003)$

[15] Mursi, M., Maker, B.H.: Basic sets of polynomials of several complex variables i. In: The Second Arab Sci. Congress, pp. 51-60. Cairo (1955)

[16] Mursi, M., Maker, B.H.: Basic sets of polynomials of several complex variables ii. In: The Second Arab Sci. Congress, pp. 61-68, Cairo (1955)

[17] Nassif, M.: Composite sets of polynomials of several complex variables. Publ. Math. Debrecen 18, 43-52 (1971)

[18] Sayyed, K.A.M., Metwally, M.S., Hassan, G.F.: Effectiveness of equivalent sets of polynomials of two complex variables in polycylinders and Faber regions. Int. J. Math. Math. Sci. 2, 529-539 (2000)

[19] Whittaker, J.M.: On series of polynomials. Quart. J. Math. Oxford 5, 224239 (1934)

[20] Whittaker, J.M.: Sur les series de base de polynomes quelconques. GauthierVillars, Paris (1949)

Z. G. Kishka and M. A. Saleem

Department of Mathematics

Faculty of Science, Sohag University

Sohag 82524, Egypt

e-mail: zanhomkishka@yahoo.com

M. A. Saleem

e-mail: abuelhassan@yahoo.com

M. A. Abul-Dahab

Department of Mathematics

Faculty of Science, South Valley University

Qena 83523, Egypt

e-mail: mamabuldahab@yahoo.com

Received: December 18, 2012.

Accepted: May 4, 2013. 